\title{
EchoGéo
}

$53 \mid 2020$

Dénominations plurielles. Quand les noms de lieux se concurrencent

\section{Dénominations plurielles : quand les noms de lieux se concurrencent!}

Introduction

Frédéric Giraut

(2) OpenEdition

Journals

Édition électronique

URL : https://journals.openedition.org/echogeo/20167

DOI : $10.4000 /$ echogeo. 20167

ISSN : 1963-1197

Éditeur

Pôle de recherche pour l'organisation et la diffusion de l'information géographique (CNRS UMR 8586)

Référence électronique

Frédéric Giraut, « Dénominations plurielles : quand les noms de lieux se concurrencent ! », EchoGéo [En ligne], 53 | 2020, mis en ligne le 25 octobre 2020, consulté le 10 août 2021. URL : http:// journals.openedition.org/echogeo/20167 ; DOI : https://doi.org/10.4000/echogeo.20167

Ce document a été généré automatiquement le 10 août 2021.

EchoGéo est mis à disposition selon les termes de la licence Creative Commons Attribution - Pas d'Utilisation Commerciale - Pas de Modification 4.0 International (CC BY-NC-ND) 


\title{
Dénominations plurielles : quand les noms de lieux se concurrencent!
}

\author{
Introduction
}

\section{Frédéric Giraut}

1 La toponymie, en tant que branche de l'onomastique (étude des noms propres) en linguistique, s'intéresse aux origines des noms, à leur signification et à leur altération. Elle produit en outre un travail considérable d'inventaire et de classification dans tous les contextes et à différentes échelles, allant de celles des microtoponymes ruraux à celle des macrotoponymes, ou noms des territoires intervenant en relations internationales. Des nomenclatures, officielles ou non, et des dictionnaires érudits ou académiques existent ainsi au niveau des villes, régions et États. Des experts au niveau international travaillent à la standardisation des noms officiels et de leurs transcriptions. Les travaux issus de la toponymie sur les noms de lieux sont utilisés en histoire et géographie pour reconstituer les archéologies du peuplement, du paysage et de l'environnement à partir des précieux indicateurs que sont les noms de lieux hérités. Enfin la linguistique prend en compte la toponymie lorsqu'elle travaille sur les paysages linguistiques. La question de la dénomination et de ses motivations, des éventuelles controverses et de leurs enjeux constitue un champ moins travaillé et apparait quant à elle comme interdisciplinaire. Nommer les lieux est en effet une opération humaine essentielle de territorialisation constitutive de la géographie politique.

2 Un courant international de toponymie politique ou critique a ainsi émergé récemment consacrant le champ de la dénomination comme un champ complémentaire des études sur les noms eux-mêmes. Il s'intéresse justement aux enjeux politiques, identitaires, et fonctionnels de la dénomination des lieux. Il est donc dédié à la production et aux changements $\mathrm{du}$ toponomascape, ou paysage toponymique, qui s'inscrit plus généralement dans le paysage linguistique. La nomination, qu'elle soit officielle ou officieuse, issue d'un processus légal ou de la pratique, peut être considérée comme une technologie sociale qui attribue aux lieux et aux territoires des fonctions et des 
références et participe de l'instauration et/ou de la révélation d'un ordre social et politique.

3 Dépassant l'usage académique de la toponymie à des fins de reconstitution du peuplement et des rapports historiques à l'environnement, l'intérêt pour la dimension géopolitique à différentes échelles de la toponymie n'est pas nouveau (Azaryahu, 1996; Monmonier, 1996 ; Zelinski, 1997 ; Guillorel, 1999; Kadmon, 2000). Mais la définition d'un cadrage problématique et l'indication de champs privilégiés ainsi que des hotspots liés s'est effectuée dans les années 2000 et 2010 (Alderman, 2008; Giraut et HoussayHolzschuch, 2008a ; Berg et Vuolteenaho, 2009 ; Rose-Redwood et al., 2010 ; Bigon, 2016 ; Puzey et Kostanski, 2016). De plus, des propositions théoriques ont été avancées pour interpréter les motivations et les pratiques de nominations, issues de la linguistique (Tent et Blair, 2007) ou des sciences politiques et notamment des approches foucaldienne, debordienne et gramscienne en termes de dispositifs, de spectacularisation et d'hégémonie (Vuolteenaho et Kolamo., 2012 ; Giraut et HoussayHolzschuch, 2016). D'importants développements par le biais d'ouvrages, de numéros spéciaux de revues ou d'articles de synthèse ont déjà été produits sur plusieurs thématiques telles que l'investissement mémoriel des noms de rues (Bulot et Veschambre, 2006 ; Rose-Redwood et al., 2018), la marchandisation des noms (Light et Young, 2014 ; Medway et Warnaby, 2014) et les noms des recompositions territoriales ou du new regionalism (Giraut et Houssay-Holzschuch, 2008b).

4 Les situations toponymiques qui font l'objet de ce dossier sont celles, nombreuses et diversifiées, où l'on constate dans les usages, la cartographie et les nomenclatures une pluralité d'appellations éventuellement contradictoires et fonctionnant sur des registres différents. Ces situations fréquentes et riches d'enjeux peuvent être interprétées à l'aune de la géographie politique et culturelle comme révélatrices de la pluralité des représentations et des pratiques, mais aussi des rapports historiques à l'espace et des éventuelles revendications sur celui-ci. Ces situations de dénominations plurielles ont jusqu'à présent retenu l'attention des chercheurs lorsqu'elles s'apparentent à des revendications territoriales concurrentes et s'inscrivent dans des conflits d'ordre géopolitique. Ainsi des travaux sont à noter sur la controverse internationale au sujet du nom de Macédoine, utilisé en Grèce et revendiqué pendant plus de 20 ans par les autorités de l'actuelle Macédoine du Nord (Tziampiris, 2012; Mavromatidis, 2010), sur la dénomination de la Mer de l'Ouest vs Mer du Japon (Short \& Dubots 2020) ou encore sur celle de la Cisjordanie (West Bank) vs Judée-Samarie (Cohen et Kliot, 1992 ; Leuenberger et Schnell, 2010). En revanche, sont largement ignorées les dénominations plurielles de lieux du quotidien : rues, carrefours repères, quartiers, villes, petites régions. Pourtant, les enjeux liés à la pluralité des noms pour de mêmes lieux dans ces situations et à ces échelles sont nombreux, riches et originaux comme nous le montre la collection d'articles que recèle le présent dossier. En effet, dans des contextes divers allant du terrain de guerre à ceux de la patrimonialisation contemporaine, de la grande ville nord-américaine à la grande ville africaine, et du monde rural français au monde rural népalais, ce sont les questions des modalités de production, d'appropriation et de revendications d'appellations multiples et potentiellement contradictoires qui sont posées. De manière générale, c'est la question de l'endonyme versus l'exonyme ou du vernaculaire versus l'officiel qui est en jeu. On verra cependant que ces oppositions binaires sont quelque peu réductrices et occultent des situations plus complexes où les hybridations ne sont pas rares. 
5 Les champs de bataille de la Première Guerre mondiale constituent une situation à la fois unique et paradigmatique de la production d'une nouvelle couche de noms de lieux à valeur fonctionnelle et symbolique dans un paysage toponymique déjà dense. Une véritable cité de la guerre rassemblant des millions de personnes sur des aménagements précaires très denses s'est constituée en quelques mois et pour plusieurs années sur un substrat rural. La mise en place de cette cité internationale appela une entreprise sans égal de dénomination temporaire mais bien réelle et constitutive de l'environnement quotidien des soldats. Cette toponymie de guerre est venue se superposer à la microtoponymie rurale en la recyclant partiellement. A l'issue $\mathrm{du}$ conflit, loin de disparaître totalement avec ses aménagements militaires, la toponymie de guerre s'est prolongée dans le paysage de la commémoration, parfois substitué à tout autre occupation de l'espace; le plus souvent une mémoire toponymique commémorative sélective s'est maintenue en combinaison avec un paysage rural qui a partiellement repris ses droits. L'article d'Alain Devos, Pierre Taborelli et Robin Perarnau porte sur les noms de tranchées et boyaux en Champagne tels qu'enregistrés sur les cartes militaires (Canevas de Tirs) en 1918. L'inventaire et la typologie réalisés rendent compte des conditions de production de ces noms par la hiérarchie militaire dans le cadre des régiments ou compagnies à partir de principes pratiques et fonctionnels militaires. Cela donne une composition hétérogène mais correspondant à un ensemble de logiques combinées. La reprise de certains éléments $\mathrm{du}$ substrat toponymique constitue une dimension pratique qui re-hiérarchise les éléments microtoponymiques originaux au profit de ceux qui acquièrent une valeur militaire stratégique. Par ailleurs, la nomenclature des noms nouveaux obéit, d'une part, à un besoin de classification pratique (même thème et même première lettre dans un secteur donné) mais, également, à des besoins d'appropriation par la troupe en se référant au patrimoine, à l'histoire et à la provenance du régiment ou de la compagnie, en célébrant également ses hauts faits ou ses martyrs. Enfin, une autre logique apparaît qui, si elle est minoritaire, n'en est pas moins importante, il s'agit de celle des dénominations vernaculaires sarcastiques ou ironiques relatives à l'ennemi ou aux conditions de vie et de morts.

6 L'article de Clément Millon porte également sur la toponymie liée à la Première Guerre mondiale ou Grande Guerre, mais cette fois, il s'agit de l'odonymie commémorative et postérieure, telle qu'enregistrée dans la nomenclature des voies et places des localités françaises. Nomination plurielle au sens où elle institue un registre mémoriel distinct reproduit dans le paysage odonymique aux côtés d'autres registres. Ainsi l'odonymie française liée à la Grande Guerre valorise principalement une date, le 11 novembre 1918, celle de l'Armistice qui marque la fin du conflit, et une valeur, la Paix, dont il est souvent difficile de distinguer la période de référence. D'autres figures sont également commémorées, deux personnalités notamment, Foch et Clémenceau, incarnant deux approches de la Nation conjuguées dans sa défense. A noter que la spécificité de ces commémorations, leur géographie et leur problématique sur la durée sont discutées en comparaison avec celles d'autres conflits majeurs et notamment la Seconde Guerre mondiale. Ainsi, le sens du corpus lié à la Grande Guerre apparaît comme en voie de disparition partielle et comme moins marqué par l'engagement humain, là où la Seconde Guerre mondiale est commémorée, outre quelques grandes figures (le Général De Gaulle, le Maréchal Leclerc, Jean Moulin), par la mémoire de la libération et de la résistance et leurs martyrs. Mais il est vrai que les relais de mémoire de la Grande Guerre fonctionnent également grâce aux très concrets et visibles monuments aux 
morts, omniprésents dans le paysage des localités françaises. Ils fonctionnent aussi dans l'odonymie par l'évocation de combats et de batailles dont il est cependant beaucoup plus difficile d'étudier les occurrences car les signifiants y sont des noms de lieux dont le signifié n'est pas toujours la bataille. On a là différentes facettes de la pluralité toponymique, non pas appliquée aux mêmes lieux désignés mais aux mêmes lieux référents.

Ghousmane Mohamed propose pour sa part un tour d'horizon de l'onomastique touarègue saharienne, particulièrement centré sur l'espace actuel du Niger. Cette plongée dans les ressorts linguistiques et sémantiques de la toponymie touarègue met l'accent sur le caractère mobile de la société nominatrice. Celle-ci déploie des techniques de dénomination pour des lieux qui ainsi prennent place à la fois dans un système socio-spatial cohérent sans être fixe, et dans un environnement réinterprété selon ses usages et les circulations. Cette toponymie est ainsi transposable (des travaux sur la projection toponymique touarègue en ville seront à ce titre bienvenus), et elle constitue un marqueur patrimonial non exclusif des espaces sahariens.

8 Également liée aux questions de patrimonialisation, mais cette fois à l'échelle planétaire, la dénomination des sites inscrits sur la Liste du Patrimoine mondial de l'Unesco est le sujet de l'article de Christophe Gauchon. On y note une grande diversité de composition et de types de références qui tentent de prouver leur valeur universelle, condition de leur inscription, et accessoirement leur valeur touristique (Néotoponymie, 2019). Mais les noms inscrits sur la Liste révèlent surtout la pluralité des échelles d'enjeux politiques de la patrimonialisation contemporaine. Ainsi, parmi les révélations de cet inventaire et de l'analyse de ses motivations, on note particulièrement une tendance à la restauration de toponymes autochtones en lieu et place d'exonymes issus de colonisations. Cela s'inscrit dans une démarche d'authenticité historique postcoloniale relative à des éléments de patrimoine culturel et naturel, mais peut aussi se combiner à une posture nationaliste lorsqu'il s'agit de promouvoir par exemple le nom népalais du toit du Monde vis à vis bien sûr du nom de son " découvreur » anglais, mais aussi de celui tibétain, plus ancien. La promotion de la référence régionale dans le cas de la Catalogne peut aussi s'apparenter à un certain nationalisme, plus évident encore lorsque la référence au nom de l'État est additionnée, comme à plusieurs reprises en Suisse, pour souligner le dépassement des localisations cantonales, mais au risque de la tautologie.

9 Darshan Karki et Miriam Wenner, en s'intéressant à l'usage d'ethnonymes comme toponymes pour désigner les territoires des Lowlands népalais, contribuent également à l'analyse de la dimension politico-identitaires de la néotoponymie. Ils montrent comment une revendication territoriale et toponymique menée par des élites au nom d'une appartenance de groupe transversale aux appartenances de langue, de religion et de castes est en contradiction avec la rhétorique nationaliste. Cependant, relevant elle aussi d'un essentialisme, quoi que stratégique (Giraut, 2017), cette revendication toponymique se heurte à la dimension excluante des dénominations ethnonymiques appliquées à la néotoponymie.

Loin des propositions de dénominations des territoires administratifs contemporains, la toponymie véhiculée par les contes et légendes, autrement dit l'oraliture, peut s'avérer, dans certains contextes, plus puissante pour promouvoir ou maintenir une toponymie et un imaginaire vernaculaires. C'est ce que montre Jean-Baptiste Bing en prenant les exemples parisiens et morvandiaux, hyperurbains et hyperruraux, issus de 
différentes pratiques de mobilisation politique, ou plutôt infrapolitique, de l'oraliture. Dans les différents cas, la toponymie révélée et mobilisée vient promouvoir des usages des lieux alternatifs à un projet (plantation de conifères) ou un aménagement (enfouissement de rivière) contestés.

11 La puissance subversive de la toponymie vernaculaire s'exprime plus encore dans la nomination des quartiers et lieux repères dans la ville africaine informelle. Gaston Ndock Ndock montre, en prenant l'exemple de Yaoundé, comment il y règne une polyphonie dans les quartiers d'urbanisation spontanée, lorsque des opérations de régularisation urbaine s'accompagnent d'une tentative d'imposition de noms de lieux officiels alors que les noms vernaculaires restent en usage. Ainsi le carrefour dénommé non sans dérision "J’ai raté ma vie », et qui par métonymie désigne un quartier, ne peut prendre le nom officiel de Nelson Mandela dans les usages. Cette commémoration officielle, décidée par les pouvoirs publics reviendrait en effet, non seulement à associer le grand homme à un lieu dont une des caractéristiques est d'avoir mauvaise réputation, mais surtout, elle gommerait le défi aux autorités que constitue le maintien d'un nom ironique qui affirme et déplore la condition marginale.

Dans la ville secondaire de Bindura, au Zimbabwe, Dorcas Zuvalinyenga souligne la diversité des dénominations de lieux en usage simultanément. Les différents corpus ont leur particularité. Si la couche officielle d'origine coloniale renvoie essentiellement à des exonymes, ou plus exactement à des référents externes, la couche officielle postcoloniale est en lien avec le régime, son histoire, son idéologie et ses réalisations affirmées, mais l'ensemble des corpus officiels partagent une orientation genrée au détriment du féminin. Les corpus vernaculaires issus de pratiques spontanées de dénominations ont pour caractéristique d'exprimer les préoccupations, y compris sous forme ironique, de groupes marginalisés, notamment jeunes, occupants à titre précaires ou encore locuteurs de langues minoritaires.

13 À Boston, ce sont trois appellations différentes qui illustrent la concurrence des registres pour la dénomination d'une "scène ouverte de consommation de drogues", autrement dit d'un secteur urbain où la vente et la consommation de drogues sont pratiquées ainsi que la prise en charge d'usagers par accès à des substituts. À l'intersection de plusieurs quartiers marqués par des dynamiques sociales contrastées, les différentes dénominations en usage pour ce secteur cherchent soit à stigmatiser, soit à valoriser, soit à occulter la "scène ouverte" qui le distingue. Cette étude de cas originale réalisée par Elsa Vivant illustre le poids de la créativité toponymique vernaculaire dans un contexte qui n'est pas celui d'une urbanisation spontanée, mais d'une tension entre prise en charge des problèmes sociaux urbains et gentrification.

Pour finir, Fabio Armand et Jean-Pierre Gerfaud traitent la question de la tautologie en toponymie dont il a déjà été question. Ils envisagent ainsi le cas des toponymes constitués de deux éléments de langues différentes, mais de même signification conceptuelle. Ici la pluralité toponymique est concentrée en de seuls noms composés. Les auteurs, en revenant sur les conditions de production linguistique de ces noms, montrent qu'ils interviennent dans le cas où un changement dans le peuplement et le statut des langues voit un ancien nom conservé mais « démotivé » (se référant alors au seul lieu mais plus au concept générique qui le décrivait) (Kristol, 2002), être complété par une référence au concept dans la langue des nominateurs. Ainsi le néotoponyme tautologique associe un ex-endonyme vernaculaire démotivé à un néonyme de même sens dans une langue véhiculaire. L'exemple du Col de la Forclaz en Haute-Savoie (on 
retrouve le même toponyme, non loin mais de l'autre côté d'une frontière internationale, dans le canton suisse du Valais), associe le nom de Col en français (langue nationale véhiculaire) à celui de Forclaz dont la terminaison est propre au dialecte francoprovencal alpin, et qui signifie originellement fourche en latin indiquant l'idée de croisement de routes dans un col en montagne. Tautologie toponymique qui dépasse l'opposition vernaculaire/officiel ou générique, et participe d'un paysage linguistique hybride témoignant du contact et de la rencontre de langues, de populations et de traditions politiques et culturelles.

L'un des enseignements les plus flagrants de cet ensemble d'études et analyses est la richesse et la puissance politique des registres vernaculaires lorsqu'ils sont en concurrence avec des registres officiels et ce, dans des contextes aussi différents que ceux des villes du Sud et des villes du Nord ou encore de hauts-lieux patrimoniaux à différentes échelles. Sur un plan théorique, les situations de pluralité toponymique viennent alimenter la remise en cause de l'opposition binaire exonyme/endonyme en soulignant la dimension relationnelle et parfois relative de ces registres (Woodman, 2012). Enfin sur un plan méthodologique la mise en évidence de ces situations nombreuses ouvre la voie à l'étude de leur expression cartographique dans un contexte de multiplication des supports cartographiques sur le geoweb (Noucher, 2020). En effet, la fin du monopole public sur l'information géographique, ou souveraineté cartographique, ouvre la voie à la promotion de corpus toponymiques éventuellement concurrents (qu'ils soient privés, vernaculaires ou officiels) dans la signalétique et sur différents supports en ligne ou embarqués.

\section{BIBLIOGRAPHIE}

Alderman D., 2008. Place, naming, and the interpretation of cultural landscapes. In Graham B. and Howard P. (ed.), The Ashgate research companion to heritage and identity. Aldershot, Ashgate Press, p. 195-213.

Azaryahu M., 1996. The power of commemorative street names. Environment \& Planning D: Society \& Space [En ligne], vol. 14, n 3, p. 311-330. DOI: https://doi.org/10.1068/d140311

Berg L. D., Vuolteenaho J. (ed), 2009. Critical Toponymies: The Contested Politics of Place Naming. Aldershot, Ashgate Press.

Bigon L. (ed.), 2016. Place Names in Africa. Colonial Urban Legacies, Entangled Histories. Cham, Springer.

Bulot T., Veschambre V. (ed.), 2006. Mots, traces et marques. Dimensions spatiales et linguistiques de la mémoire urbaine. Paris, L'Harmattan.

Cenoz J., Gorter D., 2006. Linguistic landscape and minority languages. International Journal of Multilingualism, vol. 3, $\mathrm{n}^{\circ}$ 1, p. 67-80.

Cohen, S. B., Kliot N., 1992. Place-Names in Israel's Ideological Struggle over the Administered Territories. Annals of the Association of American Geographers, vol. 82, nº 4, p. 653-680. 
Giraut F., Houssay-Holzschuch M., 2008a. Au nom des territoires ! Enjeux géographiques de la toponymie. L'Espace géographique, vol. 37, n² 2, p. 97-105.

Giraut F., Houssay-Holzschuch M., 2008b. Néotoponymie : formes et enjeux de la dénomination des territoires émergents. L'Espace politique, $\mathrm{n}^{\circ}$ 2, p. 5-12.

Giraut F., Houssay-Holzschuch M., 2016. Place Naming as Dispositif: Toward a Theoretical Framework. Geopolitics [En ligne], vol. 21, n 1, p. 1-21. DOI: https://doi.org/

$10.1080 / 14650045.2015 .1134493$

Giraut F., 2017. Frontières communautaires, ethno-régionalismes et apartheids. In Staszak J.-F. (ed.), Frontières en tous genres. Rennes, PUR, p. 81-102.

Guillorel H., 1999. Toponymie et politique. In Akin S. (ed.), Noms et re-noms : la dénomination des personnes, des populations, des langues et des territoires. Rouen, PUR-CNRS, p. 61-91.

Guillorel H. (ed.), 2008. Toponymie et politique : les marqueurs linguistiques du territoire. Bruxelles, Bruylant.

Kadmon N., 2000. Toponymy: the lore, laws, and language of geographical names. Vantage Press.

Kristol A. M., 2002. Motivation et remotivation des noms de lieux : réflexions sur la nature linguistique du nom propre. Rives nord-méditerranéennes, $n^{\circ}$ 11, p. 105-120.

Leuenberger, C., Schnell I., 2010. The politics of maps: Constructing national territories in Israel. Social Studies of Science, vol. 40, n 6, p. 803-842.

Light D., Young C., 2014. Toponymy as Commodity: Exploring the Economic Dimensions of Urban Place Names. International Journal of Urban and Regional Research [En ligne], vol. 39, n 3, p. 435-450. DOI: https://doi.org/10.1111/1468-2427.12153

Mavromatidis F., 2010. The Role of the European Union in the Name Dispute between Greece and FYR Macedonia. Journal of Contemporary European Studies, vol. 18, n 1, p. 47-62.

Medway D., Warnaby G., 2014. What's in a name? Place branding and toponymic commodification. Environment and Planning A [En ligne], vol. 46, n 1, p. 153-167. DOI: https:// doi.org/10.1068/a45571

Monmonier M., 1996. How to lie with maps. University of Chicago Press.

Noucher M., 2020. The Place Names of French Guiana in the Face of the Geoweb: Between Data Sovereignty, Indigenous Knowledge, and Cartographic Deregulation. Cartographica: The International Journal for Geographic Information and Geovisualization, vol. 55, n 1, p. 15-28.

Neotoponymy, 2019. Lascaux : du Lieu-dit à la marque globale. Note de recherche Blog scientifique Néotoponymie. URL : https://neotopo.hypotheses.org/2385

Puzey G., Kostanski L. (ed.), 2016. Names and Naming: People, Places, Perceptions and Power. Bristol, Multilingual Matters.

Rose-Redwood R. et al., 2010. Geographies of toponymic inscription: new directions in critical place-name studies. Progress in Human Geography [En ligne], vol. 34, n 4, p. 453-470. DOI: https://doi.org/10.1177/0309132509351042

Rose-Redwood R. et al. (ed.), 2018. The Political Life of Urban Streetscapes. London, Routledge.

Short J. R., Dubots L., 2020. Contesting Place Names: The East Sea/Sea of Japan naming issue. Geographical Review [En ligne]. DOI: https://doi.org/10.1080/00167428.2020.1827936 
Tent J., Blair D., 2009. Motivation for Naming: A Toponymic Typology. Technical Papers. A. N. P. Survey. Sydney, Linguistics Department.

Tziampiris A., 2012. The Macedonian name dispute and European Union accession. Southeast European and Black Sea Studies, vol. 12, n 1, p. 153-171.

Vuolteenaho J., Kolamo S., 2012. Textually Produced Landscape Spectacles? A Debordian Reading of Finnish Namescapes and English Soccerscapes. COLLeGIUM: Studies across Disciplines in the Humanities and Social Sciences, vol. 13, p. 132-158.

Woodman P. (ed.), 2012. The Great Toponymic Divide. Reflections on the definition and usage of endonyms and exonyms. Warszawa, Head Office of Geodesy and Cartography.

Zelinsky W., 1997. Along the Frontiers of Name Geography. The Professional Geographer [En ligne], vol. 49, n 4, p. 465-466. DOI: https://doi.org/10.1111/0033-0124.00092

INDEX

Thèmes : Sur le Champ - Sur le Terrain

\section{AUTEUR}

\section{FRÉDÉRIC GIRAUT}

Frédéric Giraut, frederic.giraut@unige.ch, est Professeur à l'Université de Genève. Il anime le blog https://neotopo.hypotheses.org/. Il a notamment publié :

- Giraut F., Houssay-Holzschulch M., 2017. Au-delà du toponyme, la dimension politique de la territorialisation par la nomination. In Pasquali S., Gonzalez J. (ed.), Au delà du toponyme.

Approches interdisciplinaires de la territorialité. Egypte et Méditerranée anciennes. Montpellier, ENiM. - Giraut F., Houssay-Holzschuch M., 2016. Place Naming as Dispositif: Toward a Theoretical Framework. Geopolitics, vol. 21, n 1, p. 1-21. DOI: https://doi.org/10.1080/14650045.2015.1134493 - Giraut F., 2017. Frontières communautaires, ethno-régionalismes et apartheids. In Staszak J.-F. (ed.), Frontières en tous genres. Rennes, Presses Universitaires de Rennes, p. 81-102. 